\title{
The Weibull Distribution: Reliability Characterization Based on Linear and Circular Consecutive Systems
}

\author{
M. S. Eliwa ${ }^{1}$, Medhat EL-Damcese ${ }^{2}$, A. H. El-Bassiouny ${ }^{1}$, Abhishek Tyag ${ }^{3}$, M. El-Morshedy ${ }^{4,5, *}$ \\ ${ }^{1}$ Department of Mathematics, Faculty of Science, Mansoura University, Mansoura 35516, Egypt \\ ${ }^{2}$ Mathematics Department, Faculty of Science, Tanta University, Egypt \\ ${ }^{3}$ Department of Statistics, Chaudhary Charan Singh University, Meerut 250004, India \\ ${ }^{4}$ Department of Mathematics, College of Science and Humanities in Saudi Arabia \\ ${ }^{5}$ Department of Statistics, Faculty of Science, Mansoura University, Mansoura 35516, Egypt
}

\begin{abstract}
Linear and circular consecutive models play a vital role to study the mechanical systems emerging in various fields including survival analysis, reliability theory, biological disciplines, and other lifetime sciences. As a result, analysis of reliability properties of consecutive $k-o u t-o f-n: F$ systems has gained a lot of attention in recent years from a theoretical and practical point of view. In the present article, we have studied some important stochastic and aging properties of residual lifetime of consecutive $k-o u t-o f-n: F$ systems under the condition $n-k+1, k \leq n$ and all components of the system are working at time $t$. The mean residual lifetime (MRL) and its hazard rate function are proposed for the linear consecutive $k-o u t-o f-n: F(\operatorname{lin} / \mathrm{con} / \mathrm{k} / \mathrm{n}: \mathrm{F})$ and circular consecutive $k-o u t-o f-n: F(\mathrm{cir} / \mathrm{con} / \mathrm{k} / \mathrm{n}: \mathrm{F})$ systems. Furthermore, several mathematical properties of the proposed MRL are examined. Finally, the Weibull distribution with two parameters is used as an example to explain the theoretical results.
\end{abstract}

Keywords Mean residual lifetime, Hazard rate function, Consecutive systems, Order statistics.

\section{AMS 2010 subject classifications 60E05, 62E10, 62N05}

DOI: 10.19139/soic-2310-5070-1132

\section{Introduction and methodology}

In a dynamic era today, from the perspective of developers and consumers, it is important for engineering systems such as computers, automobiles, airplanes, etc. to be highly reliable. Therefore, reliability assessment becomes a critical concern in the production (i.e. in planning, designing, and operation) of all engineering systems. While the system's components are configured in series, the system is referred to as a series system; on the contrary, when the components are arranged in parallel, the system is called a parallel system. The reliability of the series system is low (especially for a large series system) whereas the parallel system has high reliability but tends to be quite expensive. In this context, Chiang and Niu (1981) proposed a new concept of the consecutive $k-o u t-o f-n: F$ systems and demonstrated its applicability in modelling telecommunication and oil pipelines systems. Because of their high reliability and low cost, these systems have piqued the interest of many engineers and researchers. Here, it is commonly assumed that $1<k<n$, and for $k=1$, this $k$-out -of $-n: F$ system becomes a series system, and when $k=n$, it is reduced to a parallel system.

Based on the arrangement of the components, a consecutive $k$-out - of $-n: F$ system can be generally categorized as the linear consecutive $k-o u t-o f-n: F$ (denoted by lin/con $/ \mathrm{k} / \mathrm{n}: \mathrm{F}$ ) system and circular

\footnotetext{
*Correspondence to: M. El-Morshedy (Email:m.elmorshedy@psau.edu.sa). Department of Mathematics, College of Science and Humanities in Al-Kharj, Prince Sattam bin Abdulaziz University, Al-Kharj 11942, Saudi Arabia.
}

ISSN 2310-5070 (online) ISSN 2311-004X (print)

Copyright (C) 2021 International Academic Press 
consecutive $k$ - out $-o f-n: F$ (symbolized by cir/con/k/n:F) system. If a system has $n$ linearly (circularly) arranged components such that the system fails if and only if at least $\mathrm{k}$ consecutive components fail, then such a system is called lin/con/k/n:F (cir/con/k/n:F) system. For the circular systems, the components are labelled clockwise from 1 to $n$. One of the key differences between the lin/con/k/n:F and cir/con $/ \mathrm{k} / \mathrm{n}: \mathrm{F}$ systems is that in the latter case, the first and $n^{t h}$ components are somehow adjacent (or consecutive), while in the first case they are not. The example of Boland and Samaniego (2004) can be used for more clarification. Suppose we have a $2-$ out $-o f-5: F$ system. When the pair of components $[j, j+1]$ fails for any $j=1,2,3,4$ then both the linear and circular consecutive $2-o u t-o f-5: F$ system fail. However, if only the components $[5,2]$ fail then the circular $2-o u t-o f-5: F$ system fails but the corresponding linear $2-o u t-o f-5: F$ system continues to function. Therefore, for a given $k$ and $n$, the circular consecutive $k-o u t-o f-n: F$ system has generally greater failure possibilities than that of the corresponding linear consecutive $k-o u t-o f-n: F$ system and thus less reliability.

Consecutive k-out-n systems have been widely studied during the past several decades due to their large applicability in the analysis of different engineering systems. For instance, Bollinger and Salvia (1982) reported that such systems often occur in the design of integrated circuits. Shanthikumar (1985) discussed the lifetime distributions of consecutive $k-o u t-o f-n: F$ systems with exchangeable lifetimes. Chen and Hwang (1985) investigated the failure distributions of these consecutive systems under independent and identical components. Papastavridis (1989) developed lifetime distributions of circular consecutive $k-o u t-o f-n: F$ systems under exchangeable lifetimes. Aki and Hirano (1996) shown that the lifetime distribution of a consecutive $k-o u t-$ of $-n: F$ system can be expressed as a finite mixture of the distributions of order statistics of the lifetimes of components with the assumption that the lifetimes are independent and identical.

Chang et al. (2000, Ch.-3) and later Kuo and Zuo (2003, Ch.-9) studied various approaches to obtain reliabilities under different types of consecutive $k-o u t-o f-n: F$ systems. For the lifetime of these consecutive systems, Boland and Samaniego (2004) gave some stochastic ordering results by assuming that the components of the system are independent and identical. Under the exchangeable components, Navarro and Eryilmaz (2007) examined the reliability properties of consecutive $k-o u t-o f-n: F$ systems. In the same year, Eryilmaz (2007) provided some stochastic ordering results with s-independent, non-identical, and exchangeable components. Eryilmaz (2009) proposed the reliability properties of these consecutive systems under arbitrarily dependent components. Salehi et al. (2011) analyzed reliability properties of consecutive k-out-of-n systems with non-identical components lifetimes. Eryilmaz (2013) derived explicit expressions for the component importance measures for systems under exchangeable components and illustrated the results with Lomax type lifetime distribution. Recently, Goliforushania et al. (2018) discussed the mean residual life function of a generalized $k-o u t-o f-n: F$ system under different conditions, such as, when the number of components in each module is equal or unequal and when the components of the system are independent or exchangeable.

The consecutive $k-o u t-o f-n: F$ systems are an important class of coherent systems. Let $T$ represent the lifetime of a coherent system with component lifetimes $T_{1}, T_{2}, \ldots, T_{n}$, the signature of the system is defined as the probability vector $p_{1}, p_{2}, \ldots, p_{n}$ with $p_{i}=\operatorname{Pr}\left[T=T_{i: n}\right] ; i=1,2, \ldots, n$ where $T_{1: n} \leq T_{2: n} \leq \cdots \leq T_{n: n}$ are the ordered lifetimes. The signature of a coherent system has been found to be very useful for studying lifetime distributions of coherent systems. This is due to the fact that the concept of a "system signature" plays an important role in many applications, including the assessment of system reliability characteristics, and comparison of the performance of competing systems. Samaniego (2007) shown that for a coherent system having lifetime $T$ and independently and identically distributed component lifetimes $T_{1}, T_{2}, \ldots, T_{n}$ having absolute continuous distribution functions, holds $\operatorname{Pr}(T>t)=\sum_{i=1}^{n} p_{i} \operatorname{Pr}\left(T_{i: n}>t\right)$. From this result, we found that the reliability function of any coherent system can be expressed as a linear combination with non-negative coefficients of the reliability functions of $k$-out - of $-n: F$ systems, implying that the coherent system is a mixture of $k-o u t-o f-n: F$ systems. For a more detailed discussion on the signature, one can refer to Triantafillou and Koutras (2008) and Eryilmaz (2009). The conditional random variable $\left[T-t \mid T_{k: n}>t\right]$ denotes the residual lifetime of the system under the condition that at least $k$ components of the system are operational at time $t$. This conditional random variable has been thoroughly investigated in the context of coherent systems under exchangeable or 
independently and identically distributed components (see Khaledi and Shaked (2007), Bairamov (2002), Asadi and Bayramoglu (2006), Asadi and Goliforushani (2008), among others).

A critical approach for increasing a system's reliability is to build redundancy into it. A consecutive $k-o u t-$ of $-n: F$ system is one kind of such systems with redundancy. The lin/con $/ \mathrm{k} / \mathrm{n}: \mathrm{F}$ or cir/con $/ \mathrm{k} / \mathrm{n}: \mathrm{F}$ system is such a system with $n$ components that the system fails if and only if at least $k$ consecutive components in the system have failed. The reliability of lin/con/k/n:F can be written as follows

$$
R_{L}(t ; k, n)=\sum_{j=0}^{m} \sum_{i=0}^{j}(-1)^{i}\left(\begin{array}{c}
j \\
i
\end{array}\right) N(j ; k, n) R(t)^{n-j+i},
$$

where

$$
\begin{aligned}
& N(j ; k, n)=\left\{\begin{array}{cc}
0 ; & j>m \\
\left(\begin{array}{c}
n \\
j
\end{array}\right) ; & 0 \leq j \leq k-1 \\
\sum_{l=0}^{\lfloor j / k\rfloor}(-1)^{l}\left(\begin{array}{c}
n-j+1 \\
l
\end{array}\right)\left(\begin{array}{c}
n-l k \\
n-j
\end{array}\right) ; & k \leq j \leq n,
\end{array}\right. \\
& m=\left\{\begin{array}{l}
n-\left\lfloor\frac{n+1}{k}\right\rfloor+1 ; \quad n+1 \text { is a multiple of } k \\
n-\left\lfloor\frac{n+1}{k}\right\rfloor ; \quad n+1 \text { is not a multiple of } k,
\end{array}\right.
\end{aligned}
$$

and $m$ represents the maximum number of failed components that may exist in the system without causing the system to fail. But, $N(j ; k, n)$ represents the number of ways of allocating $j$ identical components into $n-j+1$ distinct urns subject to the restriction that at most $k-1$ components are placed in any one urn. The term $\left\lfloor\frac{n+1}{k}\right\rfloor$ represents the least integer number approximate to $\frac{n+1}{k}$. On the contrary, the reliability of cir/con/k/n:F can be formulated as follows

$$
R_{C}(t ; k, n)=\sum_{j=0}^{d} \sum_{i=0}^{j}(-1)^{i}\left(\begin{array}{l}
j \\
i
\end{array}\right) N_{C}(j ; k, n) R(t)^{n-j+i},
$$

where

$$
\begin{aligned}
& N_{C}(j ; k, n)=\left[\frac{n}{n-j}\right] N(j ; k, n) ; \quad 0 \leq j \leq d, \\
& d=\left\{\begin{array}{cc}
n-\left\lfloor\frac{n}{k}\right\rfloor ; & n \text { is a multiple of } k \\
n-\left\lfloor\frac{n}{k}\right\rfloor-1 ; & n \text { is not a multiple of } k,
\end{array}\right.
\end{aligned}
$$

and $n$ represents the maximum number of failed components that may exist in the system without causing the system to fail, while $N_{C}(j ; k, n)$ represents the number of ways of arranging $n$ components including $j$ failed ones in a circle such that at most $k-1$ failed ones are consecutive. Recently, due to the importance of the linear and circular consecutive systems, some authors studied these systems based on different probability models, for instance, Eliwa et al. (2020, 2021), El-Morshedy et al. (2020), Zaidi et al. (2021), among others.

Weibull distribution and its extensions or generalizations have been used effectively in different fields due to its flexibility in modelling data, and consequently, many authors still aimed to propose and study more extensions from this model because of the huge amount of data in various areas. To discuss any data, the statisticians should use a flexible approach or a proper tool to give a correct information around the data considered. The probability models always the best choice for any statisticians to predict the future as well as avoid the failures. For instance, Almamy et al. (2018), El-Morshedy and Eliwa (2019), Eliwa and El-Morshedy (2020a, 2020b), El-Morshedy et al. (2020a, 2020b, 2021a, 2021b, 2021c, 2021d), Tahir et al. (2020), Alizadeh et al. (2020), Elgohari and Yousof (2020), Alizadeh et al. (2021), Benkhelifa, L. (2021), Khan et al. (2017), among others. In this paper, we study the Weibull distribution from the consecutive systems view as an application in engineering field.

The rest of the article is organized as follows: Section 2 deals with the MRL of lin/con/k/n:F system and its hazard function, whereas the MRL and hazard function of cir/con/k/n:F system are studied in Section 3. In both Sections 2 and 3, the Weibull distribution is used to show the applicability of the developed theoretical results. A comparison between the MRL of lin/con/k/n:F and cir/con/k/n:F systems is done in Section 4. In the end, some concluding remarks are presented in Section 5. 


\section{The MRL of lin/con/k/n:F system and its hazard rate function}

In this section, we consider a $k-o u t-o f-n: F$ system with independent and identical distribution function $F(t)=1-R(t)$, where $R(t)$ is the reliability function (survival function) of each component in the system. The MRL for each component is equal to $\frac{1}{R(t)} \int_{t}^{\infty} R(x) d x$. The MRL function is used extensively in a wide variety of areas, including reliability engineering, survival analysis, and biomedical research since it represents the ageing mechanism. It is well known that the MRL function characterizes the distribution function F uniquely, since it contains all of the model's information, see, for example, Kotz and Shanbhag (1980) and Asadi and Bayramoglu (2006), Salehi et al. (2011), Goliforushania et al. (2018), and among others. Based on the definition of the MRL, as the lifetime of $k-o u t-o f-n$ system be $T_{k: n}$, then the MRL of the $k-o u t-o f-n$ system can be presented as follows

$$
\mathbf{E}\left(T_{k: n}-t \mid T_{k: n}>t\right)=\frac{1}{R_{k: n}(t)}=\int_{t}^{\infty} R_{k: n}(x) d x .
$$

Therefore, the MRL to lin/con/k/n:F, denoted by $\mathrm{H}_{\mathrm{n}, \mathrm{F}}^{\mathrm{k}}(\mathrm{t})$, is given as

$$
H_{n, F}^{k}(t)=\int_{0}^{\infty} R_{L}(x \mid t ; k, n) d x
$$

where $R_{L}(t ; k, n)$ represents the survival function of lin/con/k/n:F. So,

$$
\begin{aligned}
H_{n, F}^{k}(t) & =\sum_{j=0}^{m} \sum_{i=0}^{j}(-1)^{i}\left(\begin{array}{c}
j \\
i
\end{array}\right) N(j ; k, n) \int_{0}^{\infty} R(x \mid t)^{n-j+i} d x \\
& =\sum_{j=0}^{m} \sum_{i=0}^{j}(-1)^{i}\left(\begin{array}{c}
j \\
i
\end{array}\right) N(j ; k, n) \int_{t}^{\infty}\left[\frac{R(x)}{R(t)}\right]^{n-j+i} d x
\end{aligned}
$$

If $T_{1}, T_{2}, \ldots, T_{n}$ denotes the lifetime of the components in a lin/con/k/n:F system, where $T_{i}^{\prime} s$ are independent and identical distributed, then

$$
H_{n, F}^{k+1}(t)>H_{n, F}^{k}(t) \quad ; 0<k \leq n .
$$

To explain this, suppose that each component in the lin/con/k/n:F system has Weibull (W) distribution with survival function

$$
R(t)=e^{-\left(\frac{t}{\beta}\right)^{\alpha}} ; t, \alpha, \beta>0,
$$

and hazard rate function (HRF)

$$
h(t)=\alpha \frac{t^{\alpha-1}}{\beta^{\alpha}} ; t, \alpha, \beta>0,
$$

where $\alpha$ and $\beta$ are shape and scale parameter, respectively. It is one of the most widely utilized lifetime distributions in the area of reliability and survival analysis during the last several decades to study the observed failures of a wide variety of components and phenomena. It includes the exponential $(\alpha=1)$ and the Rayleigh distribution $(\alpha=2)$ as the particular cases. This model can be increasing, decreasing or constant HRF if $\alpha>1, \alpha<1$, and $\alpha=1$, respectively. Because of these attractive features, we have chosen the $\mathrm{W}$ model for our study. Therefore, the MRL when each component in the lin/con/k/n:F system follows W distribution can be defined as follows

$$
H_{n, F}^{k}(t)=\sum_{j=0}^{m} \sum_{i=0}^{j}(-1)^{i}\left(\begin{array}{c}
j \\
i
\end{array}\right) N(j ; k, n) e^{(n-j+i)\left(\frac{t}{\beta}\right)^{\alpha}} \int_{t}^{\infty} e^{-(n-j+i)\left(\frac{x}{\beta}\right)^{\alpha}} d x .
$$


The results of the MRL of lin/con/k/n:F system for different values of the shape parameters when $\alpha \leq 1$ are listed in Table 1.

Table 1. The MRL for lin/con/k/n:F based on the $\mathrm{W}$ model.

\begin{tabular}{l|l|l|l|l|l|l}
\hline \hline Case & $\alpha$ & $\beta$ & $n$ & $k$ & $t$ & MRL \\
\hline \hline I & 1 & 1 & 5 & 2 & 5 & 0.7000 \\
& & & & & 10 & 0.7000 \\
& & & & & 15 & 0.7000 \\
\hline II & 0.5 & 1 & 5 & 2 & 5 & 2.3438 \\
& & & & & 10 & 3.0054 \\
& & & & & 15 & 3.5130 \\
\hline \hline
\end{tabular}

From Table 1, it can be easily observed that the MRL is constant in case I as the $\mathrm{W}$ distribution reduces to exponential (E) distribution when the shape parameter $\alpha$ equals 1 where the HRF is constant. But we can see that the MRL is increasing function of $t$ in case II as the HRF is decreasing when $\alpha<1$. In the case of $\alpha>1$, we cannot get a closed form for the integration in Equation (8). Therefore, some numerical computation for different values of the shape parameter $\alpha, t$ and $k$ are listed in Table 2 .

Table 2. The MRL for (lin/con/k/n:F) based on $\mathrm{W}$ model for different values of $\alpha>1$.

\begin{tabular}{l|l|l|l|l|l|l}
\hline \hline Case & $\alpha$ & $\beta$ & $n$ & $k$ & $t$ & MRL \\
\hline \hline I & 1.5 & 1.5 & 5 & 2 & 7 & 12.51 \\
& & & & & 10 & 9.26 \\
& & & & & 15 & 8.17 \\
\hline II & 1.5 & 1.5 & 5 & 3 & 7 & 19.87 \\
& & & & & 10 & 16.10 \\
& & & & & 15 & 14.27 \\
\hline III & 2.5 & 1.5 & 5 & 2 & 7 & 14.98 \\
& & & & & 10 & 13.98 \\
& & & & & 15 & 13.71 \\
\hline IV & 2.5 & 1.5 & 5 & 3 & 7 & 21.89 \\
& & & & & 10 & 18.39 \\
& & & & & 15 & 17.95 \\
\hline \hline
\end{tabular}

From Table 2, the MRL is decreasing for $t>0$ if either $\alpha \rightarrow 1$ or $k \rightarrow 1$. Moreover, for $\alpha>1$ with fixed values for each parameter $\beta, n$ and $k$, the MRL is also decreasing for $t>0$. It is noted that $H_{n, F}^{k}(t) \propto \frac{1}{h(t)}$ when $\alpha>(<) 1$ where $h(t)$ represents HRF for each component in the system, hence we can conclude that for $\alpha \neq 1$ MRL function of lin/con $/ \mathrm{k} / \mathrm{n}: \mathrm{F}$ system is inversely proportional to the HRF of its component. The recurrence relation to MRL for lin/con/k/n:F system can be made as follows

$$
H_{n, F}^{k}(t)-H_{n, F}^{k+1}(t)=a H_{n, F}^{k-1}(t)
$$

for $k \leq n ; n, k, a>0$. The previous relation plays an important role in achieving MRL $H_{n_{1}, F}^{k+2}(t)$ when $H_{n, F}^{k}(t)$ and $H_{n, F}^{k+1}(t)$ are known. The following theorem can be used to obtain the survival function of lin/con/k/n:F system by using its HRF.

Theorem 1. Suppose $T_{1}, T_{2}, \ldots, T_{n}$ denotes the independent lifetime of the components in lin/con/k/n:F system with a common absolute continuous distribution function $F$. Then, for $k \leq n ; k, n, t>0$, the survival function $R(t)$ of lin/con/k/n:F system can be expressed as follows $R(t)=e^{-\int_{0}^{t} \psi(x) d x}$; here $\psi(x)$ is the HRF in case of 
lin/con/k/n:F system and is given by

$$
\psi(t)=\frac{\frac{d}{d t} H_{n, F}^{k}(t)+\sum_{j=0}^{m} \sum_{i=0}^{j}(-1)^{i}\left(\begin{array}{c}
j \\
i
\end{array}\right) N(j ; k, n)}{n H_{n, F}^{k}(t)+\sum_{j=0}^{m} \sum_{i=0}^{j}(-1)^{i}\left(\begin{array}{c}
j \\
i
\end{array}\right)(i-j) N(j ; k, n) \int_{t}^{\infty}\left[\frac{R(x)}{R(t)}\right]^{n-j+i} d x},
$$

for $k=1,2,3, \ldots, n$.

Proof. By differentiation Equation (5) with respect to $t$, we get

$$
\begin{aligned}
\frac{d}{d t} H_{n, F}^{k}(t)= & \sum_{j=0}^{m} \sum_{i=0}^{j}(-1)^{i}\left(\begin{array}{c}
j \\
i
\end{array}\right) N(j ; k, n)\left\{\frac{-(n-j+i)}{[R(t)]^{n-j+i}} \frac{1}{R(t)} \frac{d R(t)}{d t} \int_{t}^{\infty}[R(x)]^{n-j+i} d x\right. \\
& \left.+[R(x)]^{n-j+i} \frac{d}{d t} \int_{t}^{\infty}[R(x)]^{n-j+i} d x\right\},
\end{aligned}
$$

using $\psi(t)=\frac{f(t)}{R(t)}=\frac{-1}{R(t)} \frac{d R(t)}{d t}$, we get Equation (10).

Under the condition that each component in the system has $\mathrm{W}$ distribution when the shape parameter $\alpha \leq 1$, the following observations can be made:

1. The HRF of lin/con/k/n:F system $\psi(t)$ is constant in case of constant HRF for each component in the system. Thus, the MRL $H_{5, F}^{2}(t)$ is also constant.

2. Also, the HRF $\psi(t)$ is decreasing in case of each component in the system is decreasing HRF.

3. Similarly, in the case of increasing HRF for each component of the system, the HRF $\psi(t)$ is also increasing.

Consider $L_{1}$ and $L_{2}$ are two lin/con/k/n:F systems which have the same number of components $n$, with $R_{1}(t)>(<) R_{2}(t)$ or $h_{2}(t)>(<) h_{1}(t)$ where $R_{1}(t)$ and $R_{2}(t)$ are the survival function to $L_{1}$ and $L_{2}$, respectively and $h_{1}(t)$ and $h_{2}(t)$ are the HRF of each component in the system $L_{1}$ and $L_{2}$, respectively. Then, $H_{n_{1}, F}^{k}(t)>(<) H_{n_{2}, F}^{k}(t)$ where $H_{n_{1}, F}^{k}(t)$ and $H_{n_{2}, F}^{k}(t)$ are respectively the MRL to the first and second lin/con/k/n:F systems. Let $L_{1}$ and $L_{2}$ are two lin/con/k/n:F systems with W survival function where $R_{1}(t)<$ $R_{2}(t)$. Then, $H_{n_{1}, F}^{k}(t)<H_{n_{2}, F}^{k}(t)$. See, Table 3 .

Table 3. The MRL for two (lin/con/k/n:F)

based on the $\mathrm{W}$ model.

\begin{tabular}{l|l|l|l|l|l|l}
\hline \hline System & $\alpha$ & $\beta$ & $n$ & $k$ & $\psi(6)$ & $H_{5, F}^{2}(6)$ \\
\hline \hline$L_{1}$ & $1 / 2$ & 1 & 5 & 2 & 0.1587 & 4.1759 \\
\hline$L_{2}$ & $1 / 3$ & 1 & 5 & 2 & 0.0124 & 7.2432 \\
\hline \hline
\end{tabular}

The previous assumption can be easily verified through observations in Table 3 .

\section{The MRL of cir/con/k/n:F system and its HRF}

In this Section, based on the definition of the MRL in Equation (3), we can develop the MRL of cir/con/k/n:F system, say $M_{n, F}^{k}(t)$, as follows

$$
M_{n, F}^{k}(t)=\int_{0}^{\infty} R_{C}(x \mid t ; k, n) d x,
$$


where $R_{C}(t ; k, n)$ represents the survival function of $\operatorname{cir} / \mathrm{con} / \mathrm{k} / \mathrm{n}: \mathrm{F}$ system. So,

$$
\begin{aligned}
M_{n, F}^{k}(t) & =\sum_{j=0}^{m} \sum_{i=0}^{j}(-1)^{i}\left(\begin{array}{c}
j \\
i
\end{array}\right) N(j ; k, n) \int_{0}^{\infty} R(x \mid t)^{n-j+i} d x \\
& =\sum_{j=0}^{d} \sum_{i=0}^{j}(-1)^{i}\left(\begin{array}{c}
j \\
i
\end{array}\right) N_{C}(j ; k, n) \int_{t}^{\infty}\left[\frac{R(x)}{R(t)}\right]^{n-j+i} d x .
\end{aligned}
$$

If $T_{1}, T_{2}, \ldots, T_{n}$ denotes the lifetime of the components in a cir/con $/ \mathrm{k} / \mathrm{n}: \mathrm{F}$ system, where $T_{i}^{\prime} s$ are independent and identical distributed, then

$$
M_{n, F}^{k+1}(t)>M_{n, F}^{k}(t) \quad ; 0<k \leq n .
$$

If each component in the cir/con/k/n:F system has a W distribution, then the MRL for this system can be formulated as

$$
M_{n, F}^{k}(t)=\sum_{j=0}^{m} \sum_{i=0}^{j}(-1)^{i}\left(\begin{array}{c}
j \\
i
\end{array}\right) N_{C}(j ; k, n) e^{(n-j+i)\left(\frac{t}{\beta}\right)^{\alpha}} \int_{t}^{\infty} e^{-(n-j+i)\left(\frac{x}{\beta}\right)^{\alpha}} d x .
$$

The results of the MRL of $\operatorname{cir} / \mathrm{con} / \mathrm{k} / \mathrm{n}: \mathrm{F}$ system for different values of the shape parameter $\alpha$ when $\alpha \leq 1$ are reported in Table 4.

Table 4. The MRL for cir/con/k/n:F based on the $\mathrm{W}$ model.

\begin{tabular}{l|l|l|l|l|l|l}
\hline \hline Case & $\alpha$ & $\beta$ & $n$ & $k$ & $t$ & MRL \\
\hline \hline I & 1 & 1 & 5 & 2 & 10 & 0.8458 \\
& & & & & 15 & 0.8458 \\
& & & & & 20 & 0.8458 \\
\hline II & 0.5 & 1 & 5 & 2 & 10 & 7.7165 \\
& & & & & 15 & 8.9188 \\
& & & & & 20 & 9.9324 \\
\hline \hline
\end{tabular}

From Table 4, it is observed that the MRL is constant in case I because the HRF is constant. But, the MRL is increasing in case II because the HRF is decreasing for each component. Since we cannot get a closed form for the integration in Equation (13) or $\alpha>1$. Therefore, some numerical computation for different values of the shape parameter $\alpha, t$ and $k$ are listed in Table 5 .

Table 5. The MRL for cir/con/k/n:F based on the $\mathrm{W}$ model for different values of $\alpha>1$.

\begin{tabular}{l|l|l|l|l|l|l}
\hline \hline Case & $\alpha$ & $\beta$ & $n$ & $k$ & $t$ & MRL \\
\hline \hline I & 1.1 & 1.5 & 5 & 2 & 10 & 18.97 \\
& & & & & 15 & 18.62 \\
& & & & & 20 & 17.99 \\
\hline II & 1.1 & 1.5 & 5 & 3 & 10 & 15.32 \\
& & & & & 15 & 14.22 \\
& & & & & 20 & 13.10 \\
\hline III & 2 & 1.5 & 5 & 2 & 10 & 22.90 \\
& & & & & 15 & 22.33 \\
& & & & & 20 & 21.01 \\
\hline IV & 2 & 1.5 & 5 & 3 & 10 & 17.63 \\
& & & & & 15 & 16.22 \\
& & & & & 20 & 14.92 \\
\hline \hline
\end{tabular}

From Table 5, the MRL is decreasing for $t>0$ if either $\alpha \rightarrow 1$ or $k \rightarrow 1$. Further, for $\alpha>1$ with fixed values for each parameter $\beta, n$ and $k$, the MRL is also decreasing for $t>0$. Hence, $M_{n, F}^{k}(t) \propto \frac{1}{h(t)}$ when $\alpha>(<) 1$ where 
$h(t)$ represents the hazard rate to each component. Therefore, we can conclude that for $\alpha \neq 1$ MRL function of the $\mathrm{cir} / \mathrm{con} / \mathrm{k} / \mathrm{n}: \mathrm{F}$ system is inversely proportional to the HRF of its component. Lemma 4 has been verified for $\alpha=0.5$ and $\beta=1.5$ with $k=2,3$ as an example. The recurrence relation to the MRL for $\mathrm{cir} / \mathrm{con} / \mathrm{k} / \mathrm{n}: \mathrm{F}$ system can be made as follows

$$
M_{n, F}^{k}(t)-M_{n, F}^{k+1}(t)=b M_{n, F}^{k-1}(t),
$$

for $k \leq n ; n, k, b>0$.This relation is very important to predict the MRL $M_{n_{1}, F}^{k+2}(t)$ when $M_{n, F}^{k}(t)$ and $M_{n, F}^{k+1}(t)$ are known. The following theorem can be used to obtain the survival function $R(t)$ of $\operatorname{cir} / \mathrm{con} / \mathrm{k} / \mathrm{n}: \mathrm{F}$ system by using its $\operatorname{HRF} \varphi(t)$.

Theorem 2. Suppose $T_{1}, T_{2}, \ldots, T_{n}$ denotes the independent lifetime of the components in cir/con/k/n:F system with a common absolutely continuous distribution function $F$. Then, for $k \leq n ; k, n, t>0$, the survival function of cir/con/k/n:F can be expressed as follows $R(t)=e^{-\int_{0}^{t} \varphi(x) d x}$; here $\varphi(x)$ is the HRF in case of cir/con/k/n:F system and is given by

$$
\varphi(t)=\frac{\frac{d}{d t} M_{n, F}^{k}(t)+\sum_{j=0}^{m} \sum_{i=0}^{j}(-1)^{i}\left(\begin{array}{c}
j \\
i
\end{array}\right) N_{C}(j ; k, n)}{n M_{n, F}^{k}(t)+\sum_{j=0}^{m} \sum_{i=0}^{j}(-1)^{i}\left(\begin{array}{c}
j \\
i
\end{array}\right)(i-j) N_{C}(j ; k, n) \int_{t}^{\infty}\left[\frac{R(x)}{R(t)}\right]^{n-j+i} d x},
$$

for $k=1,2,3, \ldots, n$.

Proof. It is easy to prove this theorem by differentiation Equation (12) with respect to $t$.

Table 6 represents the HRF values for cir/con/k/n:F system when each component in this system follows W distribution with $\alpha \leq 1$.

Table 6. The MRL for cir/con/k/n:F based on the $\mathrm{W}$ model.

\begin{tabular}{l|l|l|l|l|l|l}
\hline \hline Case & $\alpha$ & $\beta$ & $n$ & $k$ & $t$ & $\phi(t)$ \\
\hline \hline I & 1 & 1 & 5 & 2 & 10 & 0.8072 \\
& & & & & 15 & 0.8072 \\
& & & & & 20 & 0.8072 \\
\hline II & 0.5 & 1 & 5 & 2 & 10 & 0.0213 \\
& & & & & 15 & 0.0146 \\
& & & & & 20 & 0.0111 \\
\hline \hline
\end{tabular}

From Table 6, the following observation can be made:

1. The HRF of cir/con/k/n:F system $\varphi(t)$ is constant in case of constant HRF for each component in the system. Thus, the MRL $M_{5, F}^{2}(t)$ is also constant.

2. Also, $\varphi(t)$ is decreasing in case of the HRF of each component in the system is decreasing.

3. Missing the line of increasing as in the case of lin/con $/ \mathrm{k} / \mathrm{n}: \mathrm{F}$ system.

4. $M_{n, F}^{k}(t) \propto \frac{1}{h(t)}$ when $\alpha>(<) 1$ where $h(t)$ represents HRF for each component in the system.

Consider $C_{1}$ and $C_{2}$ are two cir/con/k/n:F systems which have the same number of components $n$, with $R_{1}(t)>(<) R_{2}(t)$ or $h_{2}(t)>(<) h_{1}(t)$ where $R_{1}(t)$ and $R_{2}(t)$ are the survival function to $C_{1}$ and $C_{2}$, respectively and $h_{1}(t)$ and $h_{2}(t)$ are the HRF of each component in the system $C_{1}$ and $C_{2}$, respectively. Then, $M_{n_{1}, F}^{k}(t)>(<) M_{n_{2}, F}^{k}(t)$ where $M_{n_{1}, F}^{k}(t)$ and $M_{n_{2}, F}^{k}(t)$ are respectively the MRL of the first and second of cir/con $/ \mathrm{k} / \mathrm{n}: \mathrm{F}$ systems. Let $C_{1}$ and $C_{2}$ are two cir/con $/ \mathrm{k} / \mathrm{n}: \mathrm{F}$ systems with W survival function where $R_{1}(t)<$ $R_{2}(t)$. Then, $M_{n_{1}, F}^{k}(t)<M_{n_{2}, F}^{k}(t)$. See, Table 7 .

Table 7. The MRL for two cir/con/k/n:F based on the $\mathrm{W}$ model.

\begin{tabular}{l|l|l|l|l|l|l}
\hline \hline System & $\alpha$ & $\beta$ & $n$ & $k$ & $\phi(6)$ & $M_{5, F}^{2}(6)$ \\
\hline \hline$C_{1}$ & $1 / 2$ & 1 & 5 & 2 & 0.042 & 3.17 \\
\hline$C_{2}$ & $1 / 3$ & 1 & 5 & 2 & 0.037 & 10.034 \\
\hline \hline
\end{tabular}




\section{Comparative Results: MRL of lin/con/k/n:F versus MRL of cir/con/k/n:F systems}

Consider lin/con/k/n:F and cir/con/k/ n:F systems with same survival function $R(t)$ and the number of components $n$, then for $k \leq n ; n, k>0$, we observe that $M_{n, F}^{k}(t)>H_{n, F}^{k}(t)$ in case of decreasing HRF for each component, while in case of increasing HRF for each component, we get $M_{n, F}^{k}(t)<H_{n, F}^{k}(t)$. As an example, for the lin/con/2/5:F and cir/con/2/5:F systems, we can easily notice that $M_{5, F}^{2}(t)>H_{5, F}^{2}(t)$ at $\alpha=0.5<1$ and $\beta=0.9$. We can confirm the mathematical result for MRL that for a $2-$ out $-o f-5: F$ system, $M_{5, F}^{2}(t)>H_{5, F}^{2}(t)$ at $\alpha=0.5<1$ and $\beta=0.9$.

\section{Conclusion}

In this emerging technological era, it can be easily seen that there are wide applications of coherent systems in diverse engineering fields and various attractive problems are found in the structure of these systems. In this article, we have addressed consecutive $k-o u t-o f-n: F$ systems which are a special class of coherent systems. Here, we have studied the stochastic and aging properties of MRL of linear and circular consecutive $k-o u t-o f-n: F$ systems under the condition $n-k+1, k \leq n$ and all components of the system are operational at time $t$. We have developed MRL and its HRF for linear consecutive and circular consecutive systems. In addition, many interesting mathematical properties related to the MRLs of these systems such as recurrence relation for MRLs, comparison of MRLs for successive values of $k$, etc. have been obtained. Comparative results have also been provided for the MRLs of linear and circular consecutive systems for different forms of the hazard rate of system components. Finally, we have investigated the established theoretical findings by assuming that each component in these consecutive systems follows the Weibull distribution with the same parameters.

\section{Acknowledgement}

This project was supported by the deanship of scientific research at Prince Sattam bin Abdulaziz University, AlKharj, Saudi Arabia.

\section{REFERENCES}

1. Almamy, J. A., Ibrahim, M., Eliwa, M. S., Al-mualim, S., \& Yousof, H. M. (2018). The two-parameter odd Lindley Weibull lifetime model with properties and applications. International journal of statistics and probability, 7(4), 1927-7040.

2. Aki, S., \& Hirano, K. (1996). Lifetime distribution and estimation problems of consecutive-k-out-of-n: F systems. Annals of the Institute of Statistical Mathematics, 48, 185-199.

3. Aki, S., \& Hirano, K. (1996). Lifetime distribution and estimation problems of consecutive-k-out-of-n: F systems. Annals of the Institute of Statistical Mathematics, 48(1), 185-199.

4. Alizadeh, M., Afify, A. Z., Eliwa, M. S., \& Ali, S. (2020). The odd log-logistic Lindley-G family of distributions: properties, Bayesian and non-Bayesian estimation with applications. Computational Statistics, 35(1), 281-308.

5. Alizadeh, M., Khan, M. N., Rasekhi, M., \& Hamedani, G. G. (2021). A New Generalized Modified Weibull Distribution. Statistics, Optimization \& Information Computing, 9(1), 17-34.

6. Asadi, M., \& Bayramoglu, I. (2006). The mean residual life function of a k-out-of-n structure at the system level. IEEE Transactions on Reliability, 55(2), 314-318.

7. Asadi, M., \& Goliforushani, S. (2008). On the mean residual life function of coherent systems. IEEE Transactions on Reliability, 57(4), 574-580

8. Bairamov, I., Ahsanullah, M., \& Akhundov, I. (2002). A residual life function of a system having parallel or series structures. Journal of Statistical Theory and Applications, 1(2), 119-132.

9. Benkhelifa, L. (2021). The Weibull Birnbaum-Saunders distribution and its applications. Statistics, Optimization \& Information Computing, 9(1), 61-81.

10. Boland, P. J., \& Samaniego, F. J. (2004). Stochastic ordering results for consecutive k-out-of-n: F systems. IEEE Transactions on Reliability, 53(1), 7-10.

11. Bollinger, R. C., \& Salvia, A. A. (1982). Consecutive-k-out-of-n: F networks. IEEE Transactions on Reliability, 31(1), 53-56.

12. Chang, G. J. L. R., In-Hang, C., Cui, L., \& Hwang, F. K. (2000). Reliabilities of consecutive-k systems (Vol. 4). Springer Science \& Business Media.

13. Chiang, D. T., \& Niu, S. C. (1981). Reliability of consecutive-k-out-of-n: F system. IEEE Transactions on Reliability, 30(1), 87-89. 
14. Chen, R. W., \& Hwang, F. K. (1985). Failure distributions of consecutive-k-out-of-n: F systems. IEEE Transactions on Reliability, 34(4), 338-341.

15. Elgohari, H.,\& Yousof, H. (2020). New extension of weibull distribution: copula, mathematical properties and data modeling. Statistics, Optimization \& Information Computing, 8(4), 972-993.

16. Eliwa, M. S., Alshammari, F. S. S., Abualnaja, K. M., \& El-Morshedy, M. (2021). A Flexible Extension to an Extreme Distribution. Symmetry, 13(5), 745

17. Eliwa, M. S., El-Morshedy, M., \& Ali, S. (2020). Exponentiated odd Chen-G family of distributions: Statistical properties, Bayesian and non-Bayesian estimation with applications. Journal of Applied Statistics, 1-27. https://doi.org/10.1080/02664763.2020.1783520

18. Eliwa, M. S., \& El-Morshedy, M. (2020a). Bayesian and non-Bayesian estimation of four-parameter of bivariate discrete inverse Weibull distribution with applications to model failure times, football and biological data. Filomat, 34(8), 2511-2531.

19. Eliwa, M. S., \& El-Morshedy, M. (2020b). Bivariate odd Weibull-G family of distributions: properties, Bayesian and non-Bayesian estimation with bootstrap confidence intervals and application. Journal of Taibah University for science, 14(1), 331-345.

20. El-Morshedy, M., Alshammari, F. S., Hamed, Y. S., Eliwa, M. S., \& Yousof, H. M. (2021b). A new family of continuous probability distributions. Entropy, 23(2), 194.

21. El-Morshedy, M., Alshammari, F. S., Tyagi, A., Elbatal, I., Hamed, Y. S., \& Eliwa, M. S. (2021c). Bayesian and frequentist inferences on a type I half-logistic odd Weibull generator with applications in engineering. Entropy, 23(4), 446.

22. El-Morshedy, M., El-Faheem, A. A., \& El-Dawoody, M. (2020). Kumaraswamy inverse Gompertz distribution: Properties and engineering applications to complete, type-II right censored and upper record data. Plos one, 15(12), e0241970.

23. El-Morshedy, M., Eliwa, M. S., El-Gohary, A., Almetwally, E. M., \& EL-Desokey, R. (2021a). Exponentiated Generalized Inverse Flexible Weibull Distribution: Bayesian and Non-Bayesian Estimation Under Complete and Type II Censored Samples with Applications. Communications in Mathematics and Statistics, 1-22.

24. El-Morshedy, M., \& Eliwa, M. S. (2019). The odd flexible Weibull-H family of distributions: Properties and estimation with applications to complete and upper record data. Filomat, 33(9), 2635-2652.

25. El-Morshedy, M., Eliwa, M. S., \& Afify, A. Z. (2020a). The odd Chen generator of distributions: Properties and estimation methods with applications in medicine and engineering. J. Natl. Sci. Found. Sri Lanka, 48, 113-130.

26. El-Morshedy, M., Eliwa, M. S., El-Gohary, A., \& Khalil, A. A. (2020b). Bivariate exponentiated discrete Weibull distribution: statistical properties, estimation, simulation and applications. Mathematical Sciences, 14(1), 29-42.

27. El-Morshedy, M., Eliwa, M. S., \& Tyagi, A. (2021d). A discrete analogue of odd Weibull-G family of distributions: properties, classical and Bayesian estimation with applications to count data. Journal of Applied Statistics, 1-25.

28. Eryilmaz, S. (2013). Component importance for linear consecutive-k-out-of-n and m-consecutive-k-Out-of-n systems with exchangeable components. Naval Research Logistics (NRL), 60(4), 313-320.

29. Eryilmaz, S. (2007). On the lifetime distribution of consecutive k-out-of-n:F system. IEEE Transactions on Reliability, 56, 35-39.

30. Eryılmaz, S. (2009). Reliability properties of consecutive k-out-of-n systems of arbitrarily dependent components. Reliability Engineering \& System Safety, 94(2), 350-356.

31. Goliforushani, S., Xie, M., \& Balakrishnan, N. (2018). On the mean residual life of a generalized k-out-of-n system. Communications in Statistics-Theory and Methods, 47(10), 2362-2372.

32. Khaledi, B. E., \& Shaked, M. (2007). Ordering conditional lifetimes of coherent systems. Journal of Statistical Planning and Inference, 137(4), 1173-1184

33. Khan, R., Khan, M. A., \& Khan, M. A. R. (2017). Relations for moments of generalized record values from additive Weibull distribution and associated inference. Statistics, Optimization\& Information Computing, 5(2), 127-136.

34. Kotz, S., \& Shanbhag, D. N. (1980). Some new approaches to probability distributions. Advances in Applied Probability, $903-921$.

35. Kuo, W., \& Zuo, M. J. (2003). Optimal reliability modeling: principles and applications. John Wiley \& Sons.

36. Navarro, J., \& Eryilmaz, S. (2007). Mean residual lifetimes of consecutive-k-out-of-n systems. Journal of Applied Probability, 44(1), 82-98.

37. Papastavridis, S. G. (1989). Lifetime distribution of circular consecutive-k-out-of-n: F systems with exchangeable lifetimes. IEEE Transactions on Reliability, 38(4), 460-461.

38. Salehi, E. T., Asadi, M., \& Eryılmaz, S. (2011). Reliability analysis of consecutive k-out-of-n systems with non-identical components lifetimes. Journal of Statistical Planning and Inference, 141(8), 2920-2932.

39. Samaniego, F. J. (2007). System signatures and their applications in engineering reliability (Vol. 110). Springer Science \& Business Media.

40. Shanthikumar, J. G. (1985). Lifetime distribution of consecutive-k-out-of-n: F systems with exchangeable lifetimes. IEEE Transactions on Reliability, 34(5), 480-483.

41. Tahir, M. H., Hussain, M. A., Cordeiro, G. M., El-Morshedy, M., \& Eliwa, M. S. (2020). A new Kumaraswamy generalized family of distributions with properties, applications, and bivariate extension. Mathematics, 8(11), 1989.

42. Triantafyllou, I. S., \& Koutras, M. V. (2008). On the signature of coherent systems and applications. Probability in the Engineering and Informational Sciences, 22(1), 19.

43. Zaidi, S. M., Sobhi, M. M. A., El-Morshedy, M., \& Afify, A. Z. (2021). A new generalized family of distributions: Properties and applications. Aims Math, 6, 456-476. 